11

\title{
Резонансное рассеяние плоских электромагнитных волн ГГЦ диапазона кольцевыми диэлектрическими линейными структурами
}

\author{
(ㄷ А.Б. Шварцбург ${ }^{1}$, Л.М. Василяк ${ }^{1}$, С.П. Ветчинин ${ }^{1}$, К.В. Алыбин ${ }^{2}$, О.Д. Вольпян ${ }^{3}$, Ю.А. Обод ${ }^{4}$, \\ В.Я. Печеркин ${ }^{1}$, П.А. Привалов ${ }^{5}$, Д.В. Чуриков ${ }^{4}$ \\ ${ }^{1}$ Объединенный институт высоких температур РАН, \\ 125412 Москва, Россия \\ ${ }^{2}$ Акционерное общество „Научно-производственная корпорация Системы прецизионного приборостроения“, \\ 111024 Москва, Россия \\ ${ }^{3}$ Научно-производственный комплекс Фотрон-Авто, \\ 115191 Москва, Россия \\ ${ }^{4}$ Научно-технологический центр уникального приборостроения РАН, \\ 117342 Москва, Россия \\ ${ }^{5}$ МИРЭА - Российский технологический университет, \\ 119454 Москва, Россия \\ e-mail: vpecherkin@yandex.ru
}

Поступила в редакцию 08.10.2020 г.

В окончательной редакции 08.10.2020 г.

Принята к публикации 05.11.2020 г.

Исследованы обратное резонансное рассеяние на основной магнитной моде и волновые свойства линейных структур, состоящих из субволновых диэлектрических элементов в виде плоских тонких колец, возбуждаемых токами смещения падающей плоской электромагнитной волны СВЧ диапазона. Показано, что для одиночного кольца магнитное поле на основной резонансной частоте сосредоточено внутри кольца и в ближней зоне, а для структур, состоящих из двух и более колец, магнитное поле регистрируется и в дальней зоне. Измеренные основные магнитные резонансы в спектре электромагнитных полей одного и двух плоских колец совпадают с рассчитанными резонансными частотами.

Ключевые слова: метаматериалы, диэлектрический магнитный диполь, отрицательный магнитный отклик, диэлектрическое кольцо, диэлектрическая структура, плоская электромагнитная волна, резонанс.

DOI: $10.21883 /$ OS.2021.02.50560.255-20

\section{Введение}

Искусственные материалы с отрицательной магнитной $\mu<0$ и диэлектрической проницаемостями $\varepsilon<0$ обладают уникальными электродинамическими свойствами, отсутствующими у природных материалов [1-4]. В последние $10-15$ лет наблюдается стремительный рост интереса к явлениям резонансной электромагнитной индуктивности в полностью диэлектрических структурах, облучаемых электромагнитными волнами. Показано, что вариации магнитных составляющих этих волн индуцируют токи смещения в диэлектрическом материале, которые, в свою очередь, генерируют магнитные моды. Эффективность этой генерации резко усиливается, когда частоты возбуждающих волн приближаются к резонансным частотам диэлектрических структур, определяемым их формами, размерами и диэлектрическими свойствами. Анализ этих явлений составляет в настоящее время одно из новых направлений в электродинамике сплошных сред [5]. Хотя диэлектрические кольцевые резонаторы $\mathrm{GHz}$ диапазона рассматривались несколько десятилетий назад [6], систематические исследования магнитных мод стали „горячей“ темой позже в связи с открытием „искусственного магнетизма“ $[7,8]$.
Это необычное явление было продемонстрировано резонансным возбуждением магнитных мод и образованием отрицательной магнитной индуктивности в полностью диэлектрических субволновых кольцах в поле монохроматической электромагнитной волны $[9,10]$. Диэлектрические резонаторы можно рассматривать как обобщения известного колебательного контура Томсона [11], объединяющего индуктивные и емкостные свойства в одной резонансной структуре. Для диэлектрических дисков [12], цилиндров $[6,13]$ и сфер $[14,15]$ рассмотрена еще одна группа оптических эффектов намагничивания, связанных с резонансным рассеянием электромагнитных волн в теории Ми.

Электромагнитные свойства отдельных элементов являются основой для управления волновыми процессами при создании метаматериалов применительно к фундаментальным и прикладным задачам в инфракрасном (ИК) и микроволновом полях $[5,16]$. В ИК и видимом диапазонах спектра резонансные элементы в наноразмерных структурах имеют характерный размер около $100 \mathrm{~nm}$ и экспериментально могут быть измерены только некоторые усредненные электродинамические параметры. Поэтому для того, чтобы понять физическую картину явлений, необходимо дополнить ее компьютер- 
ным моделированием. Экспериментальные исследования в СВЧ диапазоне в ближней зоне позволяют выявить детализацию физических явлений на уровне отдельных субволновых элементов различной геометрии и сравнить их с теоретическими расчетами и результатами моделирования в ИК и видимом спектрах. Типы возбуждаемых резонансных мод в отдельных субволновых элементах зависят от формы резонатора, типа падающей волны, ее поляризации и ориентации в пространстве. Использование диэлектрических материалов с высоким значением диэлектрической проницаемости позволяет создавать субволновые элементы, размеры которых значительно меньше длины волны падающего излучения в СВЧ диапазоне, и переходить на более высокие частоты, в том числе ТГц и ИК диапазоны, и даже вплоть до видимых частот [5,17].

Конструкции на основе таких диэлектрических элементов позволяют управлять не только электрической, но и магнитной составляющей электромагнитного поля. Управление магнитными составляющими волнового поля с помощью диэлектрических магнитных структур, называемых оптическим магнетизмом, связано с созданием наноразмерных колебательных систем и генерацией магнитных мод. В настоящее время они используются в антенной технике, в том числе при разработке субволновых широкополосных наноантенн высокой направленности, при создании магнитных зеркал и сверхчувствительных датчиков [18-20].

Ранее мы исследовали резонансные свойства тонкого диэлектрического кольца и эллипса $[10,21,22]$, и было показано, что в случае падающей плоской электромагнитной волны наибольший резонансный отклик наблюдается на основных частотах магнитных мод, соответствующих дипольным резонансам. В случае скользящего падения плоской электромагнитной волны на диэлектрический контур основная резонансная магнитная мода характеризуется отрицательным магнитным откликом [10]. Показано, что такое кольцо действует как резонансный круговой магнитный диполь в $\mathrm{GHz}$ диапазоне частот.

Целью данной работы является экспериментальное исследование основного магнитного LC-резонанса, индуцированного в линейных структурах, состоящих из субволновых диэлектрических кольцевых элементов с высокой относительной диэлектрической проницаемостью $\varepsilon \approx 160$, плоской электромагнитной волной $\mathrm{GHz}$ диапазона, падающей перпендикулярно плоскости колец в структуре.

\section{Результаты и обсуждения}

Схема измерения магнитных полей в ближней волновой зоне структуры из диэлектрических колец приведена на рис. 1.

Генерация сигналов в заданном диапазоне частот и регистрация электрического сигнала от магнитного зонда от исследуемого объекта осуществлялось анализатором радиочастотных цепей Agilent E5071C ENA Network

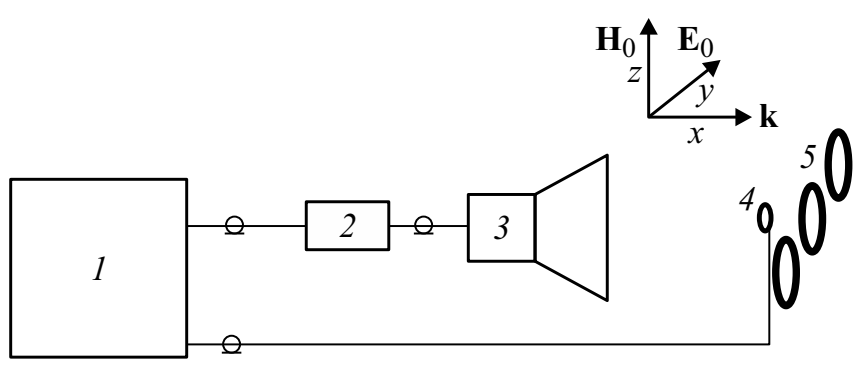

Рис. 1. Схема экспериментальной установки. 1 - анализатор радиочастотных цепей, 2 - широкополосный усилитель, 3 рупорная антенна, 4 - высокочастотный зонд магнитного поля, 5 - диэлектрические кольца.

Analyzer c рабочим диапазоном частот от $300 \mathrm{kHz}$ до $20 \mathrm{GHz}$. Плоская линейно поляризованная волна формировалась рупорной антенной (ETS-Lindgren's model 3115) с рабочим диапазоном частот $0.75-18 \mathrm{GHz}$. Для увеличения соотношения сигнал-шум и уменьшения влияния эфирных радиопомех в полосе частот $0.75-6 \mathrm{GHz}$ применялся дополнительный усилитель с коэффициентом усиления $20 \mathrm{~dB}$. Магнитные поля измерялись с помощью магнитного зонда Beehive Electronics 100B EMC Probe с внутренним диаметром кольца детектора $3.7 \mathrm{~mm}$. Плоскость кольца магнитного зонда была перпендикулярна волновому вектору $\mathbf{k}$ и параллельна векторам магнитного Н и электрического $\mathbf{E}$ полей падающей волны. В таком положении зонд не регистрирует магнитное поле падающей волны. Перед каждым испытанием проводилось измерение уровня фонового излучения с зондом при наличии падающего излучения в отсутствие тестовых объектов.

Объектами исследований являлись одиночный диэлектрический кольцевой контур и линейные структуры, состоящие из двух и трех колец одного типа. Использовались диэлектрические кольца с внешним диаметром $16 \mathrm{~mm}$ с поперечным квадратным сечением $3 \times 3 \mathrm{~mm}^{2}$. Диэлектрическая проницаемость материала колец (конденсаторная керамика) была равна $\varepsilon \approx 160$. Одиночное кольцо и структуры помещались в ближней зоне излучения антенны таким образом, чтобы векторы электрического $\mathbf{E}$ и магнитного $\mathbf{H}$ полей плоской падающей волны были параллельны плоскости кольца, а волновой вектор $\mathbf{k}$ - перпендикулярен ей (рис. 1). Целью экспериментов являлись измерения отраженных спектров рассеяния возбуждаемых магнитных полей $\mathbf{H}_{i}$ в ближней и дальней зонах одиночного диэлектрического кольца и линейноструктурируемых цепочек колец. Магнитный зонд во всех экспериментах помещался на линии оси симметрии кольца и структур относительно волнового вектора $\mathbf{k}$ c ближней к антенне стороны структур.

На рис. 2 приведены экспериментальные спектры резонансных откликов одиночного кольца в ближней и дальней зонах от кольца. На рис. 2 видно, что частота основного магнитного резонанса $f_{0}=2.48 \mathrm{GHz}$, индуцированного падающей волной, регистрируется в ближней зоне диэлектрического кольца (кривая 1). В дальней 


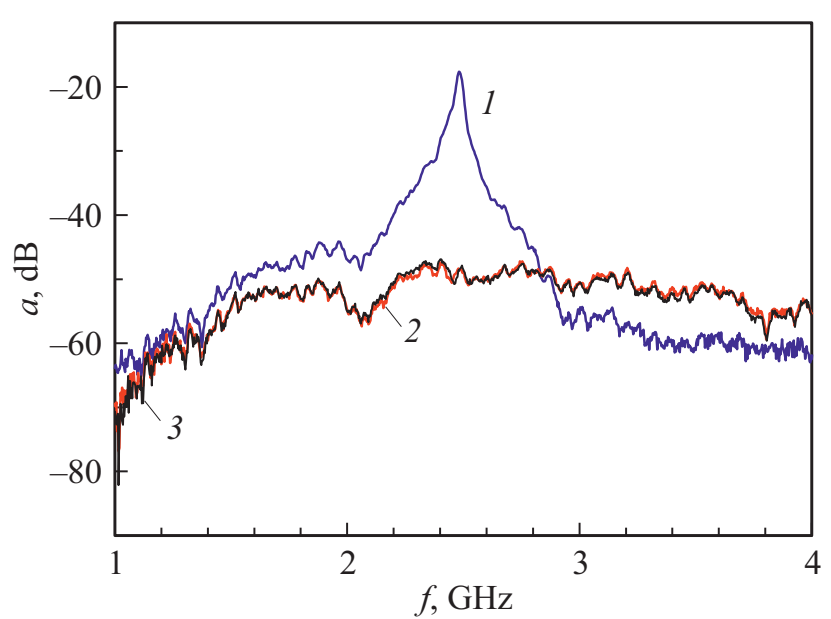

Рис. 2. Резонансные спектры индуцированного магнитного поля одного кольца. Датчик расположен в центре кольца с ближней стороны от антенны на разных расстояниях от плоскости кольца (вдоль волнового вектора, ось $x$ ): 2 (1), $30 \mathrm{~mm}(2), 3$ - спектр без кольца (сигнал фона).

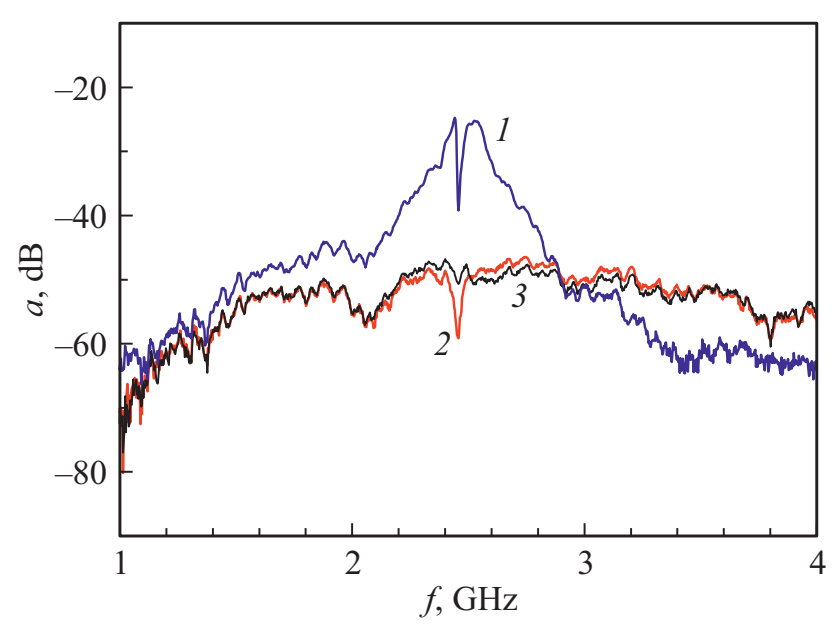

Рис. 3. Резонансные спектры индуцированного магнитного поля двух колец. Датчик расположен в центре системы двух колец с ближней стороны от антенны на разных расстояниях от плоскости колец (вдоль волнового вектора, ось $x$ ): 2 (1), $30 \mathrm{~mm}$ (2), 3 - спектр без кольца (фон).

зоне от кольца обратного рассеяния падающей волны не наблюдается (кривая 2), измеренный сигнал в области резонансной частоты совпадает с фоном (кривая 3 ).

Измеренная резонансная частота $2.48 \mathrm{GHz}$ одного кольца близка к рассчитанной резонансной частоте $2.44 \mathrm{GHz}$ при возбуждении электромагнитной плоской волной при скользящем падении в предположении однородного распределения токов смещения в поперечном сечении кольца. Некоторое отличие измеренного значения от рассчитанной величины может быть связано как с погрешностью измерения диэлектрической проницаемости керамики и изменением диэлектрической проницаемости для разных колец, так и с погрешностями расчета индуктивности кольца с квадратным сечением.
На рис. 3 приведены экспериментальные спектры резонансных откликов структуры из двух колец. Кольца были расположены вдоль оси у своими плоскостями в одной плоскости, параллельной электрическому вектору $\mathbf{E}$ (ось $y)$ и перпендикулярной волновому вектоpy $\mathbf{k}$ (ось $x$ ). Расстояние между краями колец составляло $1 \mathrm{~mm}$. Зонд был расположен в центре симметрии двух колец. На рис. 3 видно, что в ближней зоне происходит расщепление основного магнитного резонанса $f_{0-}=2.44 \mathrm{GHz}$ и $f_{0+}=2.52 \mathrm{GHz}$ (кривая 1) и в дальней зоне регистрируется резонансное излучение на основной резонансной частоте $f_{0}=2.48 \mathrm{GHz}$ (кривая 2).

Появление двух резонансных частот связано с взаимной индуктивностью колец и возможностью протекания индуцированных токов в противоположных направлениях. Появление таких резонансов для другой конфигурации двух колец и при скользящем падении плоской волны было теоретически рассмотрено нами ранее $[9,21]$. В данном случае физический механизм расщепления резонанса такой же. Принципиально новым результатом по сравнению с исследованиями $[9,21]$ является появление на частоте основного магнитного резонанса пика отраженного сигнала в дальней волновой зоне (рис. 3, кривая 2). Провал в центре резонансной кривой 1 на рис. 2 также свидетельствует об излучении энергии в дальнюю зону, поскольку частота в минимуме провала соответствует частоте пика на спектре 2 , измеренном в дальней зоне.

На рис. 4 приведены экспериментальные спектры резонансных откликов линейной структуры из трех диэлектрических колец, расположенных в ряд вдоль электрического вектора (ось $y$ ) аналогично структуре с двумя кольцами. Расстояние между краями колец равно $1 \mathrm{~mm}$. На рис. 4 видно, что в ближней зоне наблюдается дополнительное расщепление магнитного резонанса $f_{0-}$ по сравнению со структурой из двух

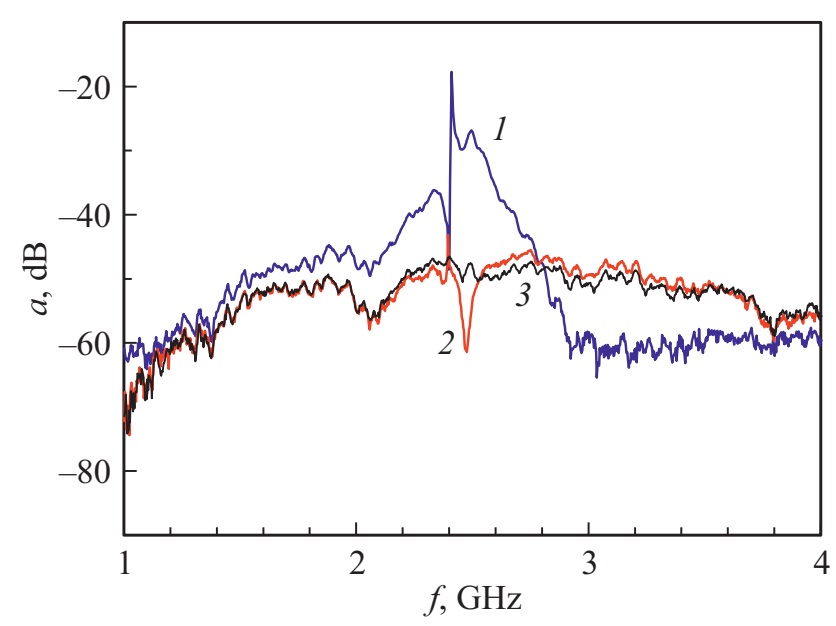

Рис. 4. Резонансные спектры индуцированного магнитного поля трех колец. Датчик расположен в центре среднего кольца с ближней стороны от антенны на разных расстояниях от плоскости кольца: 2 (1), $30 \mathrm{~mm}(2), 3$ - спектр без кольца $(ф о н)$. 
колец. Резонансная частота $f_{0-}$ понижается в этом случае до величины $2.40 \mathrm{GHz}$ и наблюдается поворот фазы на $180^{\circ}$ при незначительном повышении спектральной частоты падающей волны (рис. 4, кривая 1 ). В дальней зоне регистрируется резонансное излучение на основной резонансной частоте $f_{0}=2.48 \mathrm{GHz}$ и на расщепленной частоте $f_{0-}=2.40 \mathrm{GHz}$ (рис. 3 , кривая 2). При увеличении количества колец в линейной структуре от двух до трех амплитуда отраженного излучения в дальней зоне кольца существенно увеличилась.

\section{Выводы}

Таким образом, при нормальном падении плоской СВЧ волны на линейную структуру из диэлектрических колец, начиная с двух колец, в дальней волновой зоне от плоскости колец возникает отраженное излучение на основной частоте магнитного диполя, которое не наблюдается для одного кольца. При увеличении количества колец амплитуда отраженного сигнала на основной магнитной моде возрастает. Вследствие взаимодействия колец появляются дополнительные резонансные пики рассеянного излучения как в ближней, так и в дальней волновой зоне кольца. Количество дополнительных пиков растет с увеличением количества колец.

Обнаруженный эффект рассеяния плоской электромагнитной волны на упорядоченной системе диэлектрических колец в дальней зоне на основной резонансной частоте магнитного диполя позволит использовать такую систему для создания диэлектрических зеркал с отрицательным магнитным откликом на основном магнитном резонансе, а также на расщепленных частотах.

\section{Финансирование работы}

Работа выполнена при поддержке Министерства науки и высшего образования Российской Федерации в рамках Федеральной целевой программы „Исследования и разработки по приоритетным направлениям развития научно-технологического комплекса России на 2014-2020 годы“. Соглашение № 07515-2019-1299. Уникальный идентификатор соглашения RFMEFI60718X0206.

\section{Конфликт интересов}

Авторы заявляют, что у них нет конфликта интересов

\section{Список литературы}

[1] Veselago V.G. // Phys. Usp. 2011. V. 54. P. 1161. doi 10.3367/UFNe.0181.201111h.1201

[2] Вендик И.Б., Вендик О.Г. // ЖТФ. 2013. Т. 83. В. 10. С. 3. doi 10.1134/S1063784213010234

[3] Zhao Q., Meng Y., Du B., Kang L., Zhao H., Xie Q., Li B., Zhang X., Zhou J., Li L. // Appl. Phys. Lett. 2008. V. 92. 051106. doi 10.1063/1.2841811
[4] Bulatov M.F., Churikov D.V. // J. Surface Investigation. 2019. V. 13. N 2. P. 206. doi 10.1134/S1027451019020046

[5] Jahani S., Jacob Z. // Nat. Nanotechnol. 2016. V. 11. P. 23. doi 10.1038/nnano.2015.304

[6] Verplanken M., Van-Bladel J. // IEEE Trans. Microwave Theory and Techn. 1976. V. 24. P. 108.

[7] Miroshnichenko A.E., Kuznetsov A.I., Wei L., Fu Y., Neshev D., Luk'yanchuk B.S. Optical magnetism of dielectric nanoparticles. / Optics \& Photonics News. 2012. V. 23. N 12. P. 35. doi 10.1364/OPN.23.12.000035

[8] Kuznetsov A.I., Miroshnichenko A.E., Brongersma M.L., Kivshar Y.S., Luk'yanchuk B. // Science 2016. V. 354. P. 2472. doi $10.1126 /$ science.aag2472

[9] Jelinek L., Marques R. // J. Phys.: Condens. Matter. 2010. V. 22. P. 025902. doi 10.1088/0953-8984/22/2/025902

[10] Shvartsburg A.B., Pecherkin V.Ya., Vasilyak L.M., Vetchinin S.P., Fortov V.E. // Sci. Rep. 2017. V. 7. P. 2180. doi 10.1038/s41598-017-02310-1

[11] Pozar D. Microwave Engineering. 4th Edition. Hoboken: Wiley, 2011. $700 \mathrm{p}$.

[12] Staude I., Miroshnichenko A.E., Decker M., Fofang N.T., Liu S., Gonzales E., Dominguez J., Luk T.S., Neshev D.N., Brener I., KivsharY. // ACS Nano. 2013. V. 7. P. 7824. doi $10.1021 / \mathrm{nn} 402736 \mathrm{f}$

[13] Kapitanova P., Ternovski V., Miroshnichenko A., Pavlov N., Belov P., Kivshar Y., Tribelsky M. // Sci. Rep. 2017. V. 7. P. 731. doi 10.1038/s41598-017-00724-5

[14] Terekhov P.D., Evlyukhin A.B., Shalin A.S., Karabchevsky A. // J. Appl. Phys. 2018. V. 125. N 17. P. 173108. doi 10.1063/1.5094162

[15] Geffrin J.M., Garcia-Camara B., Gomez-Medina R., Albella P., Froufe-Perez L.S., Eyraud C., Litman A., Vaillon R., Gonzalez F., Nieto-Vesperinas M., Saenz J.J., Moreno F. // Nat. Commun. 2012. V. 3. P. 1171. doi $10.1038 /$ ncomms 2167

[16] Yang Y., Kravchenko I.I., Briggs D.P., Valentine J. // Nat. Commun. 2014. V. 5. P. 5753. doi 10.1038/ncomms6753

[17] Kuznetsov A.I., Miroshnichenko A.E., Fu Y.H., Zhang J.B., Lukyanchuk B. // Scientific Rep. 2012. V. 2. P. 492. doi 10.1038/srep00492

[18] Krasnok A.E., Maksymov I.S., Denisyuk A.I., Belov P.A., Miroshnichenko A.E., Simovski C.R., Kivshar Yu.S. // Phys. Usp. 2013. V. 56. P. 539. doi 10.3367/UFNe.0183.201306a.0561

[19] Tittl A., Leitis A., Liu M., Yesilkoy F., Choi D.Y., Neshev D.N., Kivshar Y., Altug H. // Science. 2018. V. 360. P. 1105. doi 10.1126/science.aas 9768

[20] Liberal I., Ederra I., Ramón Gonzalo, Ziolkowski R.W. // Phys. Rev. Appl. 2014. V. 1. P. 044002. doi 10.1103/PhysRevApplied.1.044002

[21] Shvartsburg A.B., Pecherkin V.Ya., Jimenez S., Vasilyak L.M., Vetchinin S.P., Vazquez L., Fortov V.E. // J. Phys. D: Appl. Phys. 2018. V. 51. N 47. P. 475001. doi 10.1088/13616463/aae1eb

[22] Shvartsburg A.B., Pecherkin V.Ya., Vasilyak L.M., Vetchinin S.P., Fortov V.E. // Phys. Usp. 2018. V. 61. P. 698. doi 10.3367/UFNe.2017.03.038139 